\title{
ARD1/NAA10 in hepatocellular carcinoma: pathways and clinical implications
}

\author{
Danbi Lee ${ }^{1}$, Myoung-Kuk Jang ${ }^{2}$, Ji Hae Seo ${ }^{3}$, Soo Hyung Ryu ${ }^{4}$, Jeong A. Kim ${ }^{5}$ and Young-Hwa Chung
}

\begin{abstract}
Hepatocellular carcinoma (HCC), a representative example of a malignancy with a poor prognosis, is characterized by high mortality because it is typically in an advanced stage at diagnosis and leaves very little hepatic functional reserve. Despite advances in medical and surgical techniques, there is no omnipotent tool that can diagnose HCC early and then cure it medically or surgically. Several recent studies have shown that a variety of pathways are involved in the development, growth, and even metastasis of HCC. Among a variety of cytokines or molecules, some investigators have suggested that arrest-defective 1 (ARD1), an acetyltransferase, plays a key role in the development of malignancies. Although ARD1 is thought to be centrally involved in the cell cycle, cell migration, apoptosis, differentiation, and proliferation, the role of ARD1 and its potential mechanistic involvement in HCC remain unclear. Here, we review the present literature on ARD1. First, we provide an overview of the essential structure, functions, and molecular mechanisms or pathways of ARD1 in HCC. Next, we discuss potential clinical implications and perspectives. We hope that, by providing new insights into ARD1, this review will help to guide the next steps in the development of markers for the early detection and prognosis of HCC.
\end{abstract}

\section{Introduction}

Arrest-defective 1 (ARD1) was originally identified as an $\mathrm{N}$-terminal acetyltransferase (NAT) that catalyzes Nterminal acetylation of proteins and has lysine acetyltransferase (KAT) activity. Various isoforms of ARD1 have been identified in mammals according to differences in amino acid number, including $\mathrm{ARD} 1^{235}, \mathrm{mARD}^{225}$, $\mathrm{ARD} 1^{198}$, and $\mathrm{ARD} 1^{131}$. Each isoform has a different biological function ${ }^{1,2}$.

ARD1 is involved in various cellular functions that regulate cell division, proliferation, and tumorigenesis. Furthermore, ARD1 is involved in normal development and viability ${ }^{3-6}$. ARD1 also appears to have a role in brain development. In particular, ARD1 plays an important role in the development of neuronal dendrites and is likely

\footnotetext{
Correspondence: Young-Hwa Chung (yhchung@amc.seoul.kr)

${ }^{1}$ Department of Internal Medicine, University of Ulsan College of Medicine,

Asan Medical Center, Seoul, Republic of Korea

${ }^{2}$ Department of Internal Medicine, Hallym University College of Medicine,

Kangdong Sacred Heart Hospital, Seoul, Republic of Korea

Full list of author information is available at the end of the article.

These authors contributed equally: Danbi Lee, Myoung-Kuk Jang, Ji Hae Seo
}

involved in neurological diseases ${ }^{7-9}$. ARD1 is also implicated in either susceptibility to or protection against oxidative stress $^{9,10}$.

Several reports have linked increased ARD1 expression to various human cancers, such as breast, prostate, lung, liver, cervical, bladder, and colorectal cancers ${ }^{11-14}$. Further, higher ARD1 expression is associated with poorer outcomes among cancer patients, including lower survival and more aggressive tumors ${ }^{11,13}$.

Hepatocellular carcinoma (HCC), which is common in Korea and representative of malignancies with a poor prognosis, is characterized by high mortality because it is typically in an advanced stage at diagnosis and because it leaves a poor hepatic functional reserve. To date, the potential role and significance of ARD1 in HCC carcinogenesis is unknown. In support of a potential link, one study showed higher ARD1 expression in HCC tissue than in non-HCC tissue, and patients with higher ARD1 expression also showed more frequent microvascular invasion (MVI) than patients with lower ARD1 expres$\operatorname{sion}^{15}$. We previously showed that ARD1 overexpression 
was associated with MVI, poor differentiation of tumors, a high cumulative recurrence rate, and poor survival ${ }^{16}$. Another report has suggested that ARD1 expression plays an important role in the development, progression, and potential recurrence of $\mathrm{HCC}^{17}$.

The present review provides a summary of recent reports regarding ARD1 and gives an overview of the essential structure, functions, and molecular mechanism or pathways of ARD1 in HCC carcinogenesis. We also discuss potential clinical implications and perspectives, such as the potential of ARD1 as a candidate tumor marker or a future target for cancer therapy.

\section{Basic concepts concerning ARD1 Discovery of ARD1}

ARD1 was first identified in Saccharomyces cerevisiae by Whiteway and Szostak in $1985^{18}$. As its full name of "arrest-defective 1" suggests, yeast ARD1 is considered to have an important role in the cell cycle and cell survival. Mutations of yeast ARD1 are associated with defects in entering the stationary phase and sporulating under nutrient-limited conditions, indicating that ARD1 is involved in controlling the switch between mitosis and alternative cell fates ${ }^{18-20}$.

ARD1 is highly conserved across organisms, from yeast to mammals. Mammalian ARD1 is located on chromosome Xq28 and regulates diverse cellular functions, including the cell cycle, cell migration, apoptosis, autophagy, differentiation, development, and diseases ${ }^{7,21-25}$. Therefore, ARD1 has garnered attention for its potential role in the development of cancer ${ }^{26-28}$. Indeed, ARD1 is upregulated in various human cancers such as lung, breast, prostate, and colorectal cancers, and its expression level is strongly associated with clinical outcomes among cancer patients ${ }^{13,14,29-31}$. Furthermore, several mechanistic studies have revealed that ARD1 directly or indirectly regulates diverse cellular pathways that are known critical steps in the progression of cancer ${ }^{21,25,32-34}$. Based on this evidence, ARD1 has emerged as a promising target for cancer therapy.

\section{ARD1 variants}

In mammal, various isoforms of ARD1 have been identified, including $\mathrm{ARD} 1^{235}, \mathrm{ARD}^{225}, \mathrm{ARD}^{198}$, and $\mathrm{ARD} 1^{131}{ }^{1,2}$. ARD1 variants are given their names based on their different numbers of amino acids; the isoforms $\mathrm{ARD} 1^{235}, \mathrm{ARD}^{225}, \mathrm{ARD} 1^{198}$, and $\mathrm{ARD} 1^{131}$ contain 235, 225,198 , and 131 amino acids, respectively. Among these variants, $\mathrm{ARD} 1^{235}$ and $\mathrm{ARD} 1^{225}$ have been most extensively characterized in human and mouse cells.

$\mathrm{ARD} 1^{235}$ and $\mathrm{ARD} 1^{225}$ isoforms share a well-conserved $\mathrm{N}$-acetyltransferase domain located between amino acids 45 and 130 (aa 45-130), although ARD1 ${ }^{131}$ and ARD1 ${ }^{198}$ have only partial $\mathrm{N}$-acetyltransferase domains. The
$\mathrm{N}$-acetyltransferase domain is considered a core region of ARD1 because it contains an acetyl-CoA-binding site (RRLGLA) located at aa 82-87 that is critical for the acetyltransferase enzyme activity of ARD1. The $\mathrm{N}$-acetyltransferase domain also contains a nuclear localization signal (NLS) (KRSHRR) located at aa 78-83, indicating a potential for dynamic translocation of ARD1 between the cytosol and the nucleus (Fig. 1a) ${ }^{1,21}$.

Whereas N-terminal domains are well-conserved in ARD1 variants, the variants have completely different amino acid sequences in C-terminal regions due to alternative RNA splicing. Alternative splicing at exon 8 alters the reading frame and introduces stop codons at amino acids 235 and 225, resulting in the production of two ARD1 isoforms, $A R D 1^{235}$ and $A R D 1^{225}$, respectively (Fig. 1b). Although nucleotide sequences of ARD1 ${ }^{225}$ are identical to those of ARD $1^{235}$ except nucleotides $472-562$ of exon $8, A R D 1^{235}$, and ARD $1^{225}$ have different amino acid sequence in their C-terminal region, starting at amino acid 158 (aa 158).

Interestingly, mouse and human cells express different types of ARD1 variants. While human cells express two ARD1 variants (i.e., $A R D 1^{235}$ and $\mathrm{ARD}^{131}$ ), mouse cells express three $\left(\mathrm{ARD} 1^{235}, \mathrm{ARD} 1^{225} \text {, and } \mathrm{ARD} 1^{198}\right)^{1}$. Thus, $\mathrm{ARD1}^{225}$ is not expressed in humans, and mouse cells express both ARD $1^{225}$ and ARD ${ }^{235}$. In contrast to mouse genomic DNA, human genomic DNA from ARD1 has no acceptor site for alternative splicing of exon 8 . Thus, the ARD1 ${ }^{225}$ transcript cannot be produced from the human genome. These data suggest that the regulation of alternative splicing of ARD1 RNA might be evolved differently between the species ${ }^{2}$.

Given the variation in amino acid sequence within Cterminal domains, it is not surprising that different ARD1 variants are associated with different functional characteristics. Indeed, while ARD $1^{225}$ inhibits angiogenesis under hypoxic conditions, ARD ${ }^{235}$ has little relationship with tumor angiogenesis ${ }^{1,35}$. Instead, ARD ${ }^{235}$ promotes cell growth under normoxia, whereas $\mathrm{ARD}^{225}$ has no effect on cell growth ${ }^{35}$. The different biological functions of ARD1 variants might reflect their different subcellular compartments. In mouse cells, $\mathrm{ARD}^{225}$ and $\mathrm{ARD} 1^{235}$ proteins are present in the cytoplasm and nucleus, respectively ${ }^{2}$. In human cells, several groups have reported the subcellular localization of $\mathrm{ARD1}{ }^{235}$; however, those results have been ambiguous and discrepant. Earlier studies reported that the majority of human ARD $1^{235}$ expression is in the nucleus, with low levels of expression in the cytoplasm ${ }^{7,21}$. By contrast, other studies suggest that human $\mathrm{ARD} 1^{235}$ is predominantly located in the cytoplasm rather than in the nucleus ${ }^{13,36}$. In light of these discrepancies, the subcellular localization of human $\mathrm{ARD} 1^{235}$ appears to differ based on the cell line, suggesting that localization of human $\mathrm{ARD} 1^{235}$ is dependent 


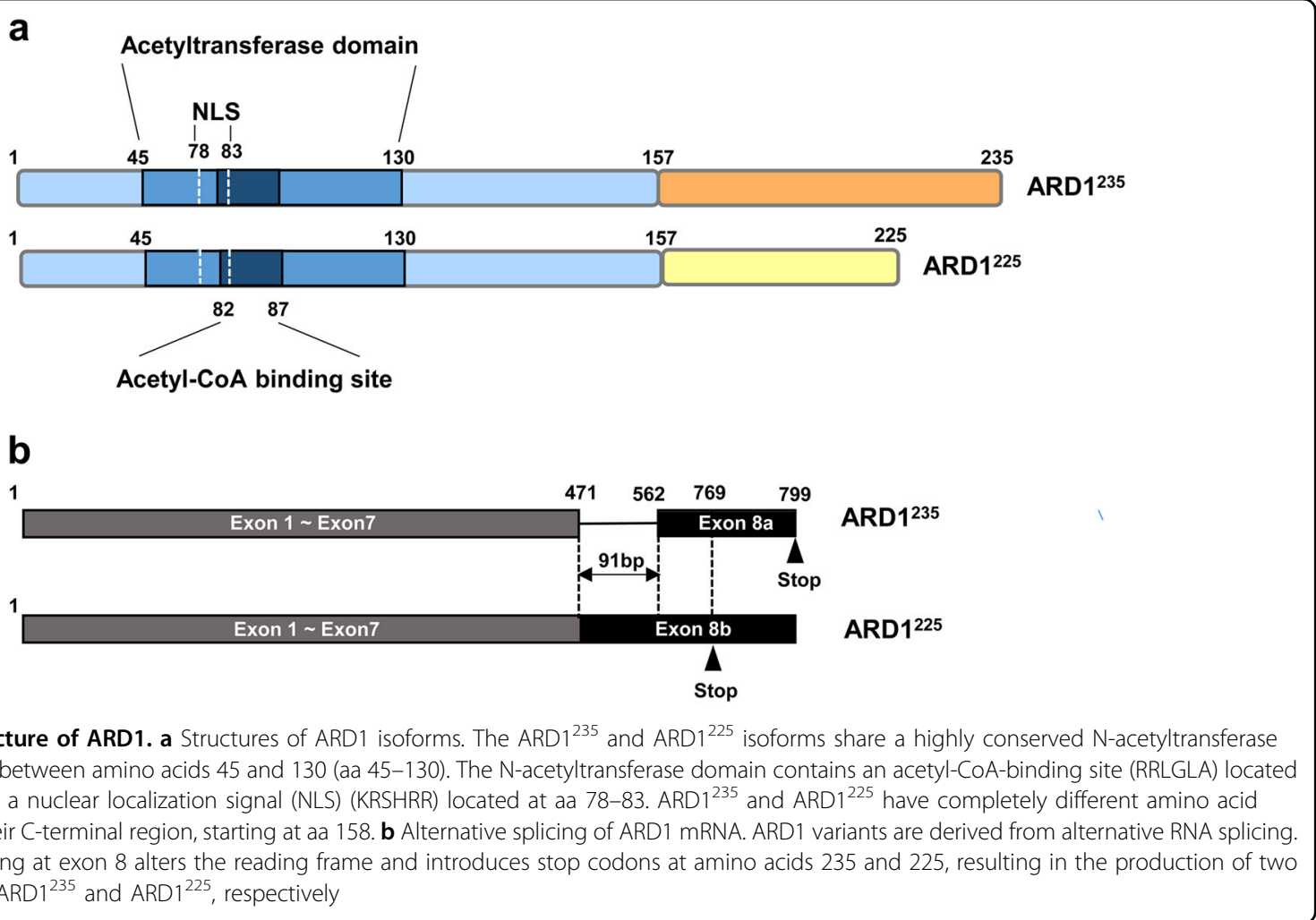

on the cellular context ${ }^{28}$. Recently, Park et al. provided one possible explanation for these complicated and often conflicting results ${ }^{37}$. They found that human ARD1 ${ }^{235}$ is imported to the nucleus of growing cells, especially during the $\mathrm{S}$ phase of the cell cycle. Furthermore, nuclear translocation of ARD $1^{235}$ is mediated by NLS, and this translocation is essential for proper cell cycle progression. Based on these results combined, ARD1 appears to have variable properties depending on subcellular localization, specific isoform, and physiological conditions. Therefore, it will be critical to consider the role of these factors in modulating ARD1 activity when evaluating the potential for cancer treatment that targets ARD1.

\section{ARD1 as an acetyltransferase}

\section{N-terminal acetyltransferase activity}

ARD1 was originally identified as a NAT that catalyzes $\mathrm{N}$-terminal acetylation of proteins ${ }^{18}$. NATs transfer acetyl groups from acetyl-CoA to the first amino acid of a given protein. $\mathrm{N}$-terminal acetylation is one of the major protein modifications in eukaryotes, occurring in $80-90 \%$ of proteins in humans and in $50-70 \%$ of proteins in yeast ${ }^{19,38}$. When proteins are synthesized and extruded from the ribosome, NATs associated with the ribosome acetylate the $\mathrm{N}$-terminal $\alpha$-amino group of the nascent polypeptide $^{39}$. Thus, protein N-terminal acetylation occurs co-translationally and irreversibly.
To date, six human NAT complexes have been identified: NatA, NatB, NatC, NatD, NatE, and NatF. The naming of each complex reflects the specific amino acid sequences contained in the $\mathrm{N}$-terminus. ARD1 is a catalytic subunit of the NatA complex that acetylates Ser, Ala, Gly, Thr, and Val within the N-terminus of a protein after an initiator methionine is cleaved by aminopeptidase ${ }^{27}$. $\mathrm{N}$-terminal acetylation is thought to play a role in protein stability by inhibiting protein degradation via blockade of $\mathrm{N}$-terminal ubiquitination ${ }^{40}$. Indeed, ARD1 acetylates the first methionine of the tuberous sclerosis 2 (TSC2) protein, which, in turn, stabilizes the TSC2 protein ${ }^{28}$. However, other studies have reported that $\mathrm{N}$-terminal acetylation does not block protein degradation. In fact, $\mathrm{N}$ terminal acetylation of a protein may even create specific degradation signals ${ }^{41}$. Beyond protein stability, several studies report other potential roles of protein $\mathrm{N}$-terminal acetylation, including protein-protein interactions and targeting subcellular localization ${ }^{42,43}$. Taken together, the existing data imply that the role of $\mathrm{N}$-terminal acetylation may differ based on the substrate protein.

Recently, N-terminal acetylation activity by ARD $1^{235}$ was found to relate strongly to genetic disease in humans. In particular, a Ser37Pro mutation of $\mathrm{ARD}^{235}$ was recently discovered in Ogden syndrome, resulting in the lethality of male infants ${ }^{44}$. Subsequent in vitro experiments have revealed that the Ser37Pro mutation 
significantly reduces the $\mathrm{N}$-terminal acetylation activity of ARD1 ${ }^{235}$, suggesting a potential link between the NAT function of ARD $1^{235}$ and human disease ${ }^{44-46}$.

\section{Lysine acetyltransferase activity}

While co-translational N-terminal acetylation is one of the most common protein modifications, a less common but potentially more important form of protein acetylation occurs post-translationally on lysine residues via KATs. The addition of an acetyl group to lysine prevents the formation of positive charges on the amino group. This modification therefore has a significant impact on the electrostatic properties of the protein. Lysine acetylation is crucial for regulating various protein functions, including chromatin-based transcriptional control, protein-protein interactions, and protein stability ${ }^{47}$.

Although ARD1 was originally identified as a NAT, its subcellular localization in non-ribosomal forms and in the nucleus strongly suggests that ARD1 has other functions. Indeed, mammalian ARD1 has both NAT and KAT activity. Mouse ARD1 $1^{225}$ was first proposed to possess KAT activity because it acetylated the lysine 532 residue of HIF- $1 \alpha$, resulting in the degradation of HIF- $1 \alpha$ via the ubiquitin-proteasome system ${ }^{48}$. Thereafter, human ARD1 ${ }^{235}$ was also shown to have KAT activity. Human $\mathrm{ARD1}^{235}$ acetylates $\beta$-catenin and is involved in the proliferation of lung cancer cells, although the target site of lysine acetylation has not yet been identified ${ }^{49,50}$. ARD1 post-translationally mediates the acetylation of a microtubule fraction purified from porcine brain in vitro ${ }^{8}$. However, the specific substrate from the microtubule fraction containing $\alpha / \beta$-tubulin has not been identified. Furthermore, ARD1 ${ }^{235}$ acetylates myosin light chain kinase (MLCK), deactivating that enzyme ${ }^{51}$. In prostate cancer cells, ARD1 acetylates the androgen receptor (AR) and this acetylation is required for AR-mediated gene transcription $^{30}$. Recent studies have uncovered substrate proteins of ARD1 and their specific target sites of lysine acetylation. In particular, during bone formation, ARD1 acetylates the lysine-225 residue of Runt-related transcription factor 2 (Runx2). Runx2 acetylation, in turn, leads to the inhibition of Runx2-mediated gene transcription, which results in the regulation of osteoblast differentiation ${ }^{52}$. Recently, Heat Shock Protein 70 (Hsp70) was identified as a new substrate of ARD1. Under stress conditions, the lysine 77 residue of Hsp70 is acetylated by ARD1, and this acetylation is essential for the enzyme activity of Hsp70 and control stress response of cells ${ }^{9}$. Furthermore, under glutamine deprivation, ARD1 acetylates the lysine-388 residue of phosphoglycerate kinase 1 (PGK1), a metabolic enzyme. PGK1 acetylation via ARD1 enhances kinase functions of PGK1 and contributes to the maintenance of cellular homeostasis in a nutrient-starved state $^{53}$. Based on evidence regarding the KAT activity of
ARD1, it is notable that ARD1 is the first identified protein with both NAT and KAT activity. However, whether KAT and NAT activity of ARD1 are regulated in similar or different ways remains unclear.

\section{Biological function of ARD1 \\ ARD1 in non-cancerous conditions}

ARD1 is involved in various cellular functions (Table 1). In particular, emerging research has shed new light on the importance of ARD1 during developmental stage. Depletion or mutation of ARD1 leads to lethality in Drosophila, Danio rerio, Caenorhabditis elegans, and Trypanosoma brucei, highlighting an essential role for ARD1 in typical development and viability ${ }^{3-6}$. Dysfunction of ARD1 is also fatal in humans, given that ARD1 mutations that cause a reduction in NAT activity can result in infant mortality, intellectual disability, development delay, and Lenz microphthalmia syndrome ${ }^{44,54-56}$. In mouse models, mice with depleted levels of the $A R D 1^{225}$ and ARD $1^{235}$ variants display severe defects in bone development ${ }^{52}$. ARD $1^{235}$ is also implicated in brain

\section{Table 1 The biological functions of ARD1}

\begin{tabular}{|c|c|c|}
\hline Category & Function & Reference \\
\hline \multirow[t]{11}{*}{ Development } & Viability of Trypanosoma brucei & Ingram et al. ${ }^{4}$ \\
\hline & Survival and proliferation & Wang et $a .^{6}$ \\
\hline & $\begin{array}{l}\text { Survival and metabolism of } \mathrm{C} \\
\text { elegans }\end{array}$ & Chen et al. ${ }^{3}$ \\
\hline & $\begin{array}{l}\text { Normal development and } \\
\text { viability of zebrafish }\end{array}$ & Ree et $a l^{5}$ \\
\hline & Ogden syndrome in males & Rope et al. ${ }^{44}$ \\
\hline & Intellectual disability & Rauch et al. ${ }^{56}$ \\
\hline & Lenz microphthalmia syndrome & $\begin{array}{l}\text { Esmailpour } \\
\text { et al. }{ }^{54}\end{array}$ \\
\hline & Global developmental delay & Popp et al..$^{55}$ \\
\hline & Bone development & Yoon et al. ${ }^{52}$ \\
\hline & Brain development & Sugiura et al. ${ }^{57}$ \\
\hline & $\begin{array}{l}\text { Neuronal dendritic } \\
\text { development }\end{array}$ & Ohkawa et al. ${ }^{8}$ \\
\hline \multirow[t]{2}{*}{ Neuronal disorders } & $\begin{array}{l}\text { Relation to neuronal } \\
\text { development }\end{array}$ & Asamui et al. ${ }^{7}$ \\
\hline & $\begin{array}{l}\text { Protection of dopaminergic } \\
\text { neurons in zebrafish }\end{array}$ & Seo et al. ${ }^{9}$ \\
\hline \multirow[t]{2}{*}{ Oxidative stress } & $\begin{array}{l}\text { Promoting oxidative injury by } \\
\text { MSRA acetylation }\end{array}$ & Shin et al. ${ }^{10}$ \\
\hline & $\begin{array}{l}\text { Maintaining protein } \\
\text { homeostasis by Hsp70 } \\
\text { acetylation }\end{array}$ & Seo et al. $^{9}$ \\
\hline
\end{tabular}


development. Mouse models suggest that $A R D 1^{235}$ is expressed in regions of cell division and migration throughout the course of brain development, and expressions of these proteins is downregulated as neurons mature and form appropriate synaptic connections ${ }^{57}$. Similarly, downregulation of ARD1 ${ }^{235}$ is observed during differentiation of neurons, suggesting that ARD ${ }^{235}$ plays an important role in development of dendrites in the brain ${ }^{8}$.

In addition to development, ARD1 is implicated in various neurological diseases. In particular, through direct interaction with amyloid- $\beta$ protein $(A \beta), A R D 1^{235}$ suppresses the secretion of $A \beta$, which is the first step in the development of Alzheimer's disease ${ }^{7}$. Another study using a zebrafish model of Parkinson's disease showed that ectopic expression of dominant-negative human ARD1 ${ }^{235}$ exacerbated dopaminergic neuronal loss and locomotor deficits compared with wild-type human ARD $1^{235}$ expression ${ }^{9}$. These results suggest that ARD ${ }^{235}$ may be protective against the development of neurodegenerative disease.

Recent studies have revealed that ARD1 is also involved in cellular response to oxidative stress. ARD $1^{235}$ represses the enzymatic activity of methionine sulfoxide reductase A, an enzyme that protects cells from oxidative stress by removing reactive oxygen species. In support of a prooxidant role of $\mathrm{ARD}^{235}$, an ARD1 ${ }^{235}$ transgenic mouse is more susceptible to oxidative stress and shows more severe tissue injury compared with wild-type mouse ${ }^{10}$. However, results of a recent study suggest the opposite effect-that $\mathrm{ARD} 1^{235}$ may be protective against oxidative stress. In this study, ARD $1^{235}$ prevented protein aggregation under oxidative stress by regulating chaperone function of Hsp70 required for the maintenance of protein homeostasis. The authors therefore concluded that ARD $1^{235}$ protects cells against oxidative stress and functions as an anti-oxidant protein ${ }^{9}$. Although it is presently unclear whether ARD1 has a protective or apoptotic role under oxidative stress, existing data suggest that ARD1 is a potential target for ameliorating oxidative stress in diverse human health conditions, such as cancer, neurological disease, inflammation, and metabolic disease.

\section{ARD1 in cancerous conditions}

Role of ARD1 as a tumor suppressor Mammalian ARD1 was first reported to act as a tumor suppressor, given that mouse ARD ${ }^{225}$ inhibits HIF- $1 \alpha$ stabilization and angiogenesis by acetylating the lysine- 532 residue $^{48}$. In contrast to $\mathrm{ARD} 1^{225}, \mathrm{ARD}^{235}$ has no effect on HIF- $1 \alpha$ acetylation or stability. However, a crucial role of ARD ${ }^{225}$ in HIF- $1 \alpha$ stability was confirmed using a mouse tumor model ${ }^{11}$. In that study, the authors generated $\mathrm{Apc}^{\mathrm{Min} /+}$ /ARD1 ${ }^{225}$ transgenic mice and demonstrated the antitumor effect of ARD $1^{225}$ in mice. In particular, they found a significant decrease in the quantity of intestinal polyps among $\mathrm{Apc}^{\mathrm{Min} /+} / \mathrm{ARD}^{225}$ transgenic mice compared with $\mathrm{Apc}^{\mathrm{Min} /+}$ transgenic mice. Within the intestinal polyps of $\mathrm{Apc}^{\mathrm{Min} /+} / \mathrm{ARD}^{225}$ transgenic mice, they found that VEGF expression was also reduced, which, in turn, suppressed tumor angiogenesis and growth.

In addition to $\mathrm{ARD} 1^{225}$, human $\mathrm{ARD} 1^{235}$ is also reported to have tumor suppressor properties. In breast cancer, higher $\mathrm{ARD}^{235}$ expression levels are correlated with better clinical outcome from the patients patients, including higher survival, smaller tumor volume, and lower rates of lymph node metastasis ${ }^{34,58}$. In lung and gastric cancers, ARD1 ${ }^{235}$ expression is significantly downregulated in malignant tumors with lymph node metastasis compared with primary tumors without metastasis, suggesting that downregulation of ARD1 plays a causal role in tumor development ${ }^{29}$.

Investigations into the underlying molecular mechanisms have led to new insights into mechanisms through which ARD1 reduces proliferation and metastasis. In breast cancer cells, inhibition of $\mathrm{ARD}^{235}$ induces autophagy by inhibiting mTOR signaling and subsequently reduces cancer cell growth ${ }^{58}$. Yi et al. reported that $\mathrm{ARD} 1^{235}$ is required for caspase activation during apoptosis and that depletion of ARD $1^{235}$ may thus provide robust protection against DNA damage-induced cell death $^{25,59}$. These data suggest a pro-apoptotic role of $\mathrm{ARD1}^{235}$. Regarding cell motility, ARD1 ${ }^{235}$ directly acetylates the lysine 608 residue of MLCK, leading to the inhibition of MLCK activity required for cell contraction and migration ${ }^{51}$. In lung cancer cells, $\mathrm{ARD}^{235}$ binds to $\mathrm{p} 21$-activated kinase-interacting exchange factor to block its downstream Rac1/Cdc42 pathway. Blockade of the Rac1/Cdc42 pathway results in the inhibition of cancer cell metastasis, although this effect appears to be independent of the acetyltransferase activity of $\mathrm{ARD}^{235}{ }^{29}$. In breast cancer cells, ARD1 $1^{235}$ inhibits cancer cell migration by targeting signal transducer and activator of transcription 5a (STAT5a) independently of acetyltransferase activity-independent manner ${ }^{34}$. Taken together, although ARD1 is an acetyltransferase enzyme, these results suggest that $\mathrm{ARD} 1^{235}$ can exert oncogenic functions, independent of its catalytic activity.

Role of ARD1 as an oncogene Although several studies have demonstrated that ARD1 is an important molecule involved in cancer progression, it is still under debate whether human $\mathrm{ARD}^{235}$ serves as an oncogene or a tumor suppressor. In fact, a growing number of studies have highlighted potential oncogenic properties of ARD1 in cancer progression (Fig. 2). At present, it is widely accepted that $\mathrm{ARD1}^{235}$ relates positively to cellular growth. Fisher et al. first suggested that $\mathrm{ARD}^{235}$ is required to maintain division and proliferation of liver 


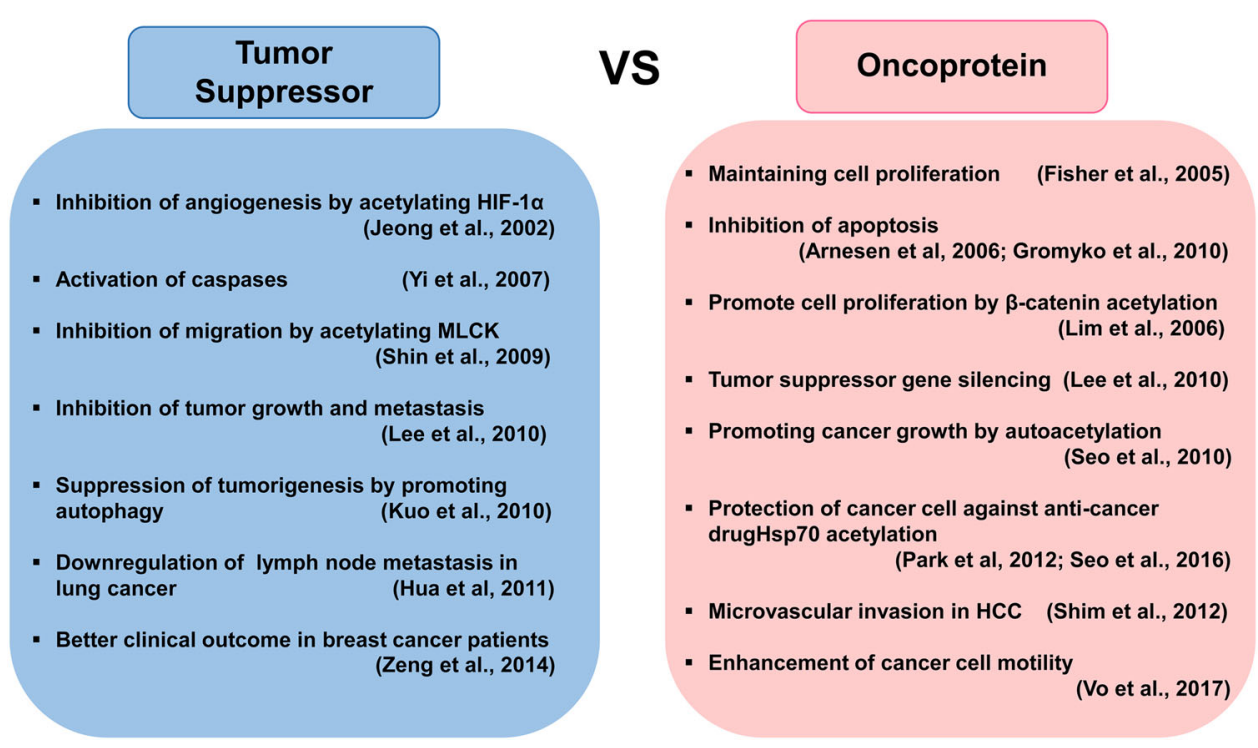

Fig. 2 Controversial roles of ARD1 in cancer development and progression. Many studies have reported an important role of ARD1 in cancer progression. However, it is still debated whether human ARD1 serves as an oncogene or a tumor suppressor

cancer cells under hypoxia ${ }^{60}$. Furthermore, a fluorescence-activated cell sorting analysis revealed that $\mathrm{ARD} 1^{235}$ promotes cell proliferation by regulating the G1/ $\mathrm{S}$ and $\mathrm{G} 2 / \mathrm{M}$ checkpoints of the cell cycle in breast and lung cancer cells, indicating that ARD $1^{235}$ is relevant for cell proliferation ${ }^{14,32,50}$. Regarding the molecular mechanisms, ARD1 ${ }^{235}$ promotes tumor growth by enhancing cyclin D1 expression, and these effects are mediated by the activation of $\beta$-catenin and activator protein $1^{32,50}$. Recently, SAM domain and HD domain-containing protein 1 -the latter of which is involved in the regulation of intracellular dNTP pools required for DNA replication -were found to be involved in $\mathrm{ARD} 1^{235}$-mediated cancer cell growth ${ }^{61}$.

Indeed, a number of links have been found between increased expression of $\mathrm{ARD} 1^{235}$ and human cancers, such as breast, prostate, lung, liver, cervical, bladder, and colorectal cancers ${ }^{12-14,62}$. In addition, high levels of $\mathrm{ARD} 1^{235}$ expression correspond with poorer outcomes among cancer patients, such as low survival rate and more aggressive tumors ${ }^{13,62}$. These point to the potential role of $\mathrm{ARD}^{235}$ as an oncoprotein.

In addition, ARD $1^{235}$ seems to promote the invasive properties of cancer cells. In lung cancer cells, ARD1 downregulates E-cadherin by regulation of DNA methyltransferase $1^{62}$. In breast cancer cells, ARD $1^{235}$ enhances cell motility by activation of Aurora A kinase function ${ }^{33}$. Based on these results, ARD $1^{235}$ expression is closely related to MVI in HCC patients ${ }^{15}$.

Other studies demonstrate that ARD $1^{235}$ has a protective effect against cell death. Depletion of ARD1 ${ }^{235}$ induces the transcription of p53-dependent pro- apoptotic genes and activation of the caspasedependent apoptosis pathway ${ }^{2,63}$. Depletion of $\mathrm{ARD}^{235}$ also revokes doxorubicin-but not tumor necrosis factor $\alpha$ (TNF $\alpha)$-induced activation of the nuclear transcription factor- $\mathrm{kB}(\mathrm{NF}-\mathrm{\kappa} B)^{64}$. Neuroblastoma cells expressing dominant-negative $\mathrm{ARD} 1^{235}$ are more sensitive to etoposide compared with wild-type ARD1-expressing cells ${ }^{9}$. These results suggest that $A R D 1^{235}$ is involved in drug-resistant mechanisms of cancer cells. Thus, ARD1 ${ }^{235}$ could be a potential marker for the prediction of chemoresistance in cancer patients. In-depth studies are needed to address the association between ARD1 ${ }^{235}$ expression and drug sensitivity or resistance of cancer patients and to elucidate the detailed molecular mechanism by which ARD $1^{235}$ protects cancer cells against anti-tumor drugs.

\section{General consideration of ARD1 in clinical respects}

HCC is the malignancy with the poorest prognosis among the most common types of cancers ${ }^{65}$. In line with this poor prognosis, the majority of $\mathrm{HCC}$ patients are hardly candidates for the best curative option, curative surgical resection, because of frequent vascular invasion and/or intrahepatic or extrahepatic metastases at the time of diagnosis ${ }^{66}$. Poorly reserved underlying liver function and frequent postoperative recurrence of $\mathrm{HCC}$ represent additional hindrances to successful disease management ${ }^{67}$. To expand the understanding of potential clinical applications of ARD1, now we are going to enter into the clinical implications of ARD1, which will be focused in a field of HCC. 
Basic clinical concepts of ARD1 in hepatocarcinogenesis

HCC is typically characterized by hypervascularity. Hypervascularity may be due to the fact that HCC typically develops in hypoxic environments caused by underlying chronic liver diseases, such as liver cirrhosis. In most HCCs, underlying chronic liver diseases necessarily provoke an alteration (i.e., decrement) in intrahepatic blood flow, which results in poor oxygen supply (i.e., hypoxia) to the liver. This hypoxia accelerates the production of angiogenic factors, including VEGF, basic fibroblast growth factor, and insulin-like growth factor-II (IGF-II). Overexpression of angiogenic factors subsequently promotes neovascularization, which further contributes to the development of HCC and leads to increased vascular density in tumors (i.e., hypervascular tumors). At the same time, overexpression or hyperactivation of angiogenic factors can enhance the growth of HCC and also promote intrahepatic or extrahepatic metastases ${ }^{68}$.

In previous studies, we sought to identify markers for the early detection and prediction of post-treatment prognosis among HCC patients using serological or histological features. Although we demonstrated their clinical significance and found their usefulness in clinical practice, the many ways to the destination mean that there is no single, perfectly effective way to cover all of them. Thus, it is essentially impossible to understand all forms of hepatocarcinogenesis and to cure HCC completely by conquering a sole pathway because it is too complex $^{68,69}$. Indeed, we found that several factors are involved in hepatocarcinogenesis, including IGF-II, TNF$\alpha$, transforming growth factor beta 1 (TGF- $\beta$ ), osteopontin, metastatic tumor antigen (MTA) 1, and MTA2. These markers can be used not only for diagnosis/prognosis but also as therapeutic targets for postoperative adjuvant therapies ${ }^{70-80}$.

A separate line of work suggests that hypoxia is associated with tumorigenesis. In particular, HIF- $1 \alpha$ has been reported to play a critical role in hepatocarcinogenesis ${ }^{81}$. In HCC, intratumoral HIF- $1 \alpha$ and VEGF expression significantly increase to promote new vessel formation by stimulating transcription of cytokines, which are involved in angiogenesis ${ }^{82}$. Relying on various conditions, HIF- $1 \alpha$ reacts differently according to the oxygen concentration in the tissues ${ }^{83}$. Under normoxia, HIF- $1 \alpha$ typically has a short half-life of $5 \mathrm{~min}$; thus, it is immediately degraded into biologically inactive products. That is, under normoxia, the proline residues at P402 and P564 of the ODD domain of HIF- $1 \alpha$ undergo hydroxylation, then ubiquitination by the von Hippel-Lindau (pVHL) protein, followed by degradation via a proteasome. At this step, the ODD domain of HIF- $1 \alpha$ plays an important role in degradation as well as the prevention of its overexpression $^{84-86}$. In contrast to normoxia, hypoxia stabilizes HIF- $1 \alpha$ to accelerate the development, growth, and subsequent metastasis of cancers via stimulating the transcription of angiogenic factors (e.g., VEGF) ${ }^{87}$. These results provide a theoretical basis for notion that the HIF$1 \alpha$ pathway is a promising target for cancer therapies ${ }^{88}$.

As a first step, it may be worth briefly mentioning the association between HIF- $1 \alpha$ and MTA1 in hepatocarcinogenesis. For more in-depth discussion, we refer the reader to previous papers ${ }^{80,89,90}$. Based on our prior studies, we found that MTA1 is more highly overexpressed in hepatitis B virus (HBV)-associated HCC than in non$\mathrm{HBV}$-associated $\mathrm{HCC}^{76}$. This may be due to the $\mathrm{HBx}$ protein of HBV that can induce MTA1 expression and result in the enhancement of hypoxia signaling via HIF- $1 \alpha$ stabilization. Consequently, stabilization of HIF- $1 \alpha$ accelerates angiogenesis under hypoxia by reducing the binding of pVHL protein to HIF- $1 \alpha$ and preventing the ubiquitin-dependent degradation of HIF-1 $\alpha$. Furthermore, MTA1 itself may increase the transcription and stabilization of HIF- $1 \alpha^{80}$. MTA1 activation by TGF- $\beta 1$ is also involved in tumor progression through the promotion of epithelial-mesenchymal transition (EMT) $)^{91,92}$.

On the other hand, it seems that the association between HIF- $1 \alpha$ and ARD1 in human HCC is not yet clear. In the acetylation process, ARD1 which exists in all mammalian cells including brain, heart, bone marrow, and liver, acts as a key molecule. ARD1 was named because its deletion impairs cell mitosis and arrests cell division. In particular, ARD1 is detected in high concentrations in tumor cells of colorectal and breast cancers in some patients ${ }^{1,21}$. Furthermore, concentrations of ARD1 are high in organs that are highly vascular (e.g., heart, liver), and thus, ARD1 may play an important role in determining the destiny of cells during cell cycles. It, therefore, is thought of ARD1 that it should be deeply involved in the progression of malignancies as the cell division increases.

The existing data, in vitro, suggest that mouse ARD1 plays a pivotal role as a negative controller of HIF- $1 \alpha$ stability under normoxia ${ }^{48,93}$. It is assumed that mouse ARD1 acetylates HIF- $1 \alpha$ protein by direct reaction with Lys $_{532}$ of the ODD domain. This deepens the instability of HIF- $1 \alpha$ and then suppresses the expression of angiogenic factors such as VEGF, which lead to be able to prevent development and/or progression of malignancies by inhibiting neovascularization. On the other hand, recent reports suggest that human ARD1 may have no impact on the stability of HIF- $1 \alpha^{36,94}$. In addition, there is a view that the ARD1 is not regulated by hypoxia in several human tumor cell lines including cervical adenocarcinoma, fibrosarcoma, and breast cancer cells.

Notably, as mentioned before, HCC is a typical hypervascular tumor and also it has been developed generally in hypoxic condition such as liver cirrhosis. Additionally, the HIF- $1 \alpha$ signaling pathway is the representative crucial 
mechanism in hepatocarcinogenesis and angiogenesis. Although, to date, the biologic functions of ARD1 have not been clearly defined in malignancies, especially HCC, and the direct association of ARD1 with HIF- $1 \alpha$ in HCC of human is not also clarified, we still wonder whether ARD1 will have a direct or indirect relationship with HIF$1 \alpha$ in human hepatocarcinogenesis.

On the basis of the facts described above, it is possible that ARD1 is also implicated in the development of HCC and its progression, including tumor growth and metastasis. Therefore, it is very valuable to investigate ARD1 expression and to ascertain its potential association with clinical characteristics for clarifying hepatocarcinogenesis and setting up the preventive strategies in the future. At the same time, it will be useful to amend current treatment modalities and develop new effective therapies.

\section{ARD1 expression and its clinical significance in non-HCC neoplasia}

Dose ARD1 act as an oncoprotein or a tumor suppressor in human cancers? This point remains to be clarified, but a number of in vitro studies on the molecular mechanisms of carcinogenesis have been published in favor of the oncoprotein role ${ }^{27,80,89,90}$. Research on ARD1 in clinical settings, however, remains in its very early stages. In fact, there are very few reports on ARD1 using human neoplastic tissues. An even smaller number of studies examined the potential association between ARD1 expression and recurrence or survival in patients with cancers $^{13-17,95-98}$. These studies are summarized in Table 2.

Yu et al. investigated human ARD1 expression in cancer tissues and compared this expression to that in nonneoplastic tissues ${ }^{14}$. For this study, the investigators enrolled a relatively large number of total subjects ( 400 with common cancers vs. 133 with matching noncancerous tissues). Across the sample, the rates of ARD1-positive tissue were $52.3 \%(209 / 400)$ of cancer tissues and $31.5 \%$ (42 of 133) of non-cancerous tissues. Although the overall difference in ARD1-positive rates between cancer and non-cancerous tissues reached statistical significance $(p<0.005)$, ARD1 was still detected in

Table 2 Clinical studies of ARD1 overexpression in malignancies including hepatocellular carcinoma in humans

\begin{tabular}{|c|c|c|c|}
\hline & Subjects & Methods of evaluation & Key findings \\
\hline Yu et al. ${ }^{14}$ & $\begin{array}{l}19 \text { common types of cancers }(n \\
=400)^{\mathrm{a}}\end{array}$ & Immunohistochemistry & $\begin{array}{l}\text { ARD1 had a significantly positive correlation with } \\
\text { common cancers overall, including individual breast and } \\
\text { colorectal cancers }\end{array}$ \\
\hline Ren et al. ${ }^{13}$ & Colorectal cancer $(n=50)$ & Immunohistochemistry & $82 \%(41 / 50)$ were ARD1 positive \\
\hline $\begin{array}{l}\text { Jiang } \\
\text { et al. }{ }^{96}\end{array}$ & Colorectal cancer $(n=270)$ & $\begin{array}{l}\text { ELISA using anti-ARD1A antibody (serum) } \\
\text { and immunohistochemistry (tissue) }\end{array}$ & $\begin{array}{l}\text { Anti-ARD1A antibody can be detected in serum from } \\
\text { patients with colon cancer. ARD1A is a novel tumor- } \\
\text { associated antigen and a prognostic factor for colon } \\
\text { cancer }\end{array}$ \\
\hline Liu et al. ${ }^{97}$ & $\begin{array}{l}\text { Colon adenocarcinoma }(n= \\
\text { 225) }\end{array}$ & Immunohistochemistry & $\begin{array}{l}\text { When combined with other tumor markers, ARD1 } \\
\text { increased the overall detection accuracy and prognostic } \\
\text { value }\end{array}$ \\
\hline $\begin{array}{l}\text { Wang } \\
\text { et al. }^{98}\end{array}$ & $\begin{array}{l}\text { Breast cancer ( } n=356: 82 \\
\text { malignant vs. } 274 \text { benign) }\end{array}$ & Immunohistochemistry & $\begin{array}{l}\text { ARD1 overexpression is correlated with breast cancer } \\
\text { and especially with lymph node metastasis and estrogen } \\
\text { receptor positivity }\end{array}$ \\
\hline $\begin{array}{l}\text { Huang } \\
\text { et al. }\end{array}$ & $\mathrm{HCC}(n=112)$ & $\begin{array}{l}\text { Microarray for single-nucleotide } \\
\text { polymorphisms (SNPs) }\end{array}$ & $\begin{array}{l}\text { Loss of heterozygosity in ARD1B was associated with } \\
\text { significantly decreased survival }\end{array}$ \\
\hline $\begin{array}{l}\text { Shim } \\
\text { et }^{15}{ }^{15}\end{array}$ & $\mathrm{HCC}(n=94)$ & Real-time PCR (mRNA) & $\begin{array}{l}\text { Intratumoral ARD1 mRNA levels were associated with } \\
\text { microvascular invasion but not with } 5 \text {-year recurrence- } \\
\text { free or overall survival }\end{array}$ \\
\hline Lee et al. ${ }^{16}$ & $\operatorname{HCC}(n=698)$ & Immunohistochemistry & $\begin{array}{l}\text { ARD1 overexpression was closely associated with } \\
\text { frequent postoperative recurrence and poor survival }\end{array}$ \\
\hline $\begin{array}{l}\text { Kang } \\
\text { et al. }{ }^{17}\end{array}$ & $\mathrm{DN}(n=51), \mathrm{HCC}(n=226)$ & Immunohistochemistry & $\begin{array}{l}\text { ARD1 expression reflects the malignant potential of } \\
\text { dysplastic hepatic nodules }\end{array}$ \\
\hline
\end{tabular}

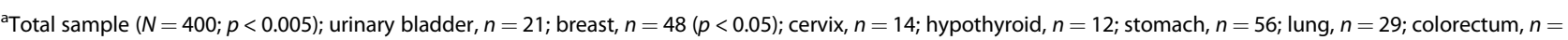
$125(p<0.01)$; prostate, $n=12$; nasopharynx, $n=11$ ( $p<0.1)$; liver, $n=9$; esophagus, $n=19$; skin, $n=14$; kidney, $n=10(p<0.1)$; gallbladder, $n=4$; eye, $n=3$; vocal cords, $n=5(p<0.1)$; pancreas, $n=2$; ovary, $n=3$; brain, $n=3$ 
a substantial number of non-cancerous tissues (i.e., $31.5 \%$ ), and this rate was not significantly different from specific cancer types (refers to footnote of Table 2). This is because only small number of cancer cases of each type were merged into the same overall group and then analyzed as a whole. Across the cancer types, there was a significant association between ARD1 overexpression and colorectal $(n=125,48.0 \%$ vs. $18.2 \% ; p<0.01)$ and breast cancers $(n=48,62.5 \%$ vs. $38.1 \% ; p<0.05)$. At any rate, the study confirmed that ARD1 expression is widespread in various cancers and, at the very least, showed its close association with colorectal and breast cancers. A link between ARD1 expression and colorectal cancers is consistent with a previous study by Ren et $\mathrm{al}^{13}$. In that study, the authors used immunohistochemistry and compared ARD1 expression in colorectal cancer and colitis tissues. They found that ARD1 was positive in 41 out of 50 (82\%) tested colorectal cancer tissues, but weakly positive in only 12 of $50(24 \%)$ matched normal tissues $(p<0.001)$ and negative in all of 20 colitis tissues $(p<0.001)$. Importantly, they showed the possibility of ARD1 as a potential biomarker in patients with colorectal cancer or even other malignancies. In fact, in the next study by the same investigators, they acquired the result with ARD1 overexpression in $84.1 \%$ (227 out of 270 ) of tested colon cancer tissues and, strikingly, successfully isolated the autoantibody against ARD1 in serum obtained from patients $^{96}$. They suggested for the first time that ARD1 is a novel tumor antigen in colon cancers and also has a potential to predict prognosis in the patients with colon cancer. In a subsequent study, the investigators used four autoantibodies (synuclein-gamma (SNCG), a human homolog of piwi (Hiwi), phosphatase of regenerating liver-3 (PRL-3), and ARD1) from 225 colon cancer patients, which were all isolated from serum, to evaluate whether the combination of them improve the values as tumor markers in terms of early detection and prognostic prediction of cancer ${ }^{97}$. Results showed that the combination of autoantibodies was associated with an elevated positive tumor marker rate $(76.9 \%$ or $173 / 225)$ and an increased detection accuracy of cancer, with $61.9 \%$ (252/ 407) for SNCG and $82.6 \%$ (336/407) for the combination, with a hazard ratio $3.2(p<0.001)$ for poor prognosis. It is somewhat conclusive that ARD1 is clearly overexpressed and can be considered a tumor-specific antigen in colon cancer.

In terms of other malignancies, fewer studies have been reported, for which reason it is difficult to conclude whether ARD1 has a potential clinical role. Consistent in part with the present conditions, ARD1 is overexpressed in malignant breast neoplasia compared with benign conditions, e.g., fibroadenomas, hyperplasia, inflammatory breast diseases, and cysts. According to a study by $\mathrm{Yu}$ et al., the ARD1-positive rate was significantly higher in breast cancer tissues than in non-cancerous ones $(62.5 \%$ $(30 / 48)$ vs. $38.1 \%(8 / 21) ; p<0.05)^{14}$. Their findings are comparable to the ARD1-positive rate reported by Wang et al. in invasive ductal carcinomas $(61.0 \%$ or $50 / 82)$ compared with benign conditions listed above $(48.8 \%$ or $119 / 244)^{98}$. Although they demonstrated the implications of ARD1 in malignant breast neoplasia, there were some limitations. Above all, the staining intensity was not graded, and the tissues of substantial proportion of benign diseases also stained positive for ARD1, which did not make ARD1 a suitable indicator for differentiating breast cancers from non-neoplastic diseases, i.e., a tumorspecific marker. Further, there was a report in contrast to it that ARD1 is a tumor suppressor in breast cancer by reducing cell proliferation and autophagy via inhibiting mTOR signaling pathway ${ }^{58}$. In thyroid cancer, as well, it remains inconclusive whether ARD1 is an oncogenic protein or a tumor suppressor ${ }^{21,99}$.

\section{ARD1 expression and its clinical significance in HCC}

So far, to our knowledge, there has been only one clinical study showing ARD1 overexpression by immunohistochemistry in human HCC samples ${ }^{14}$. However, the subjects with HCC were composed of only a very small portion of the total study population $(<3 \%, 9 / 400)$; therefore, positive rates of ARD1 detection could not be statistically compared between the HCC and non-HCC groups [33.3\% (3/9) vs. $0 \%(0 / 1), p=$ not applicable]. Also, the analyses for clinical outcomes could not be done in HCC because there were no clinical data about the patients such as HCC stages or the patients' survival. Although another microarray study showed the association of a specific single-nucleotide polymorphism (loss of heterozygosity in ARD1B) with clinicopathological characteristics and survival in HCC patients, it could not directly demonstrate the significance of ARD1 at the protein levels for the development, progression, and/or prognosis of HCC.

Recently, in light of the potential of ARD1 as a predictor of clinical outcomes, we published an important paper using $94 \mathrm{HCC}$ patients ${ }^{15}$. In the study, we hypothesized that ARD1 is overexpressed in HCC but not in non-HCC and thus may predict patients' clinical outcomes in terms of recurrence and survival. The theoretical basis has been sufficiently described above: the main feature of HCC is hypoxia in the central lesion, resulting in hypervascularity, which is mediated by HIF1- $\alpha$ pathway. In the study, we quantified ARD1 mRNA levels in HCC and matching non-HCC tissues using real-time PCR. Based on these results, patients were regrouped into high $(n=38)$ and low levels $(n=56)$ of ARD1 expression. We found that the high-expression group had more frequent MVI than the low-expression group (32\% vs. $14 \% ; p<0.05)$. Regrettably, however, we could not see the findings that 
we expected in 5 -year recurrence-free ( $34 \%$ vs. $46 \%, p=$ $0.98)$ and overall survival rates ( $76 \%$ vs. $73 \%, p=0.52)$. Importantly, intratumoral ARD1 mRNA levels were significantly high in HCCs with MVI, suggesting novel potential links between ARD1 overexpression and the clinical courses of HCC patients.

Very importantly, in April 2017, we presented novel findings from two large-scale clinical studies on the significance of ARD1 in HCC at the 52nd Annual Meeting of the European Association for the Study of the Liver in Amsterdam, the Netherlands. In the first study, results clearly demonstrated ARD1 overexpression in HCC, and a good correlationship between ARD1 expression and postoperative recurrence and patient survival. We enrolled 698 histologically confirmed HCC patients (162 with liver transplantation and 536 with curative surgical resection). By grading the intensity of ARD1 immunohistochemical staining, we investigated the association of ARD1 with the histological and radiological characters of the tumors, as well as patient clinical outcomes. The results demonstrated that ARD1 was stainable in 87\% (605/698) of tumors, and, as expected, ARD1 overexpression was positively associated with MVI $(p<0.001)$ and poor differentiation of the tumors $(p<0.001)$. Interestingly, the 5-year cumulative recurrence rates of HCC increased as the intensity of ARD1 expression in HCC tissues increased $(p<0.05)$. Further, the survival of the patients also decreased with higher ARD1 expression $(p<$ $0.01)^{16}$. In another presentation, we showed that ARD1 expression may reflect the malignant potential of dysplastic nodules, a considerable portion of which subsequently leads to $\mathrm{HCC}^{17}$. In brief, 63\% (32/51) were stained positive for ARD1 in dysplastic nodules, whereas 93\% (210/226) of HCC were stained positive ARD1 and all normal livers tested were negative ARD1 (0/4). Importantly, in the subgroups of patients histologically defined as having low-grade or high-grade dysplasia, both ARD1positive rate and its staining intensity were significantly higher in high-grade dysplastic nodules than in low-grade ones. Altogether, we found that higher intensity of ARD1 staining via immunohistochemistry is associated with higher malignant potential, even in the dysplastic stages. Taken together, the evidence suggests that ARD1 in the liver may play an important role both in the early stages of HCC development and in its progression to the advanced stages.

\section{Clinical prospective of ARD1 in HCC}

It is established that ARD1 widely exists in mammalian cells and acts as an acetyltransferase by catalyzing protein acetylation, which serves to regulate cell division, proliferation, and even tumorigenesis ${ }^{11}$. All reviewed data are in accordance with clinical observations from all colorectal cancers and selected groups with breast neoplasia as summarized in Table 2. First, what we wish to emphasize here is that ARD1 overexpression in the liver continuously changes in a stepwise pattern from low-grade dysplasia, to high-grade dysplasia to $\mathrm{HCC}$, as determined histologically. Moreover, intensity of ARD1 expression is well correlated with HCC recurrence and patient longterm survival. This means that ARD1 not only takes part in early development (i.e., from dysplasia to cancer) but also plays a key role in tumor progression (i.e., poor differentiation and MVI) and even its recurrence.

A number of obstacles still lie ahead in the study of ARD1. To use the understanding of the clinical significance of ARD1 overexpression in HCC in a clinical practice, it should be applicable for early detection (as a specific tumor marker), and curative or postoperative adjuvant treatment (as a therapeutic target). These goals are promising, given recent research findings by our group and others. In addition to other molecules (e.g., MTA1), ARD1 can be considered as a potential target in hepatocarcinogenesis and conquering HCC in the future.

\section{Conclusion}

Since ARD1 was first identified in 1985, its potential roles have been discussed constantly. ARD1 has several isoforms and plays different roles in different species. In humans, we suggest that ARD1 has specific roles in cellular growth, differentiation, neuronal development, and cancer development/progression. In particular, ARD1 may play opposite roles-as a tumor suppressor or an oncoprotein-in the tumorigenesis of different cancer types. On the basis of our recent studies, however, ARD1 might be an oncogenic protein in hepatocarcinogenesis. Furthermore, we have revealed that ARD1 is a useful diagnostic and/or prognostic index in patients with HCC. Nonetheless, the molecular signaling pathway used by ARD1 in HCC has not been completely determined. Further investigations should continue to clarify the precise molecular mechanism of ARD1 in hepatocarcinogenesis.

\section{Acknowledgements \\ This work was supported by Basic Science Research Program through the National Research Foundation of Korea (NRF) funded by the Ministry of Education (NRF-2016R1A6A1A03011325, No 2016R1D1A1B03935560), and by a National Research Foundation of Korea (NRF) grant funded by the Korean Government (MSIP) (No. 2014R1A5A2010008). \\ Author details \\ ${ }^{1}$ Department of Internal Medicine, University of Ulsan College of Medicine, Asan Medical Center, Seoul, Republic of Korea. ${ }^{2}$ Department of Internal Medicine, Hallym University College of Medicine, Kangdong Sacred Heart Hospital, Seoul, Republic of Korea. ${ }^{3}$ Department of Biochemistry, Keimyung University School of Medicine, Daegu, Republic of Korea. ${ }^{4}$ Department of Internal Medicine, Inje University College of Medicine, Seoul Paik Hospital, Seoul, Republic of Korea. ${ }^{5}$ DNA Link Inc., Seoul, Republic of Korea}

Conflict of interest

The authors declare that they have no conflict of interest. 


\section{Publisher's note}

Springer Nature remains neutral with regard to jurisdictional claims in published maps and institutional affiliations.

Received: 28 March 2018 Accepted: 11 April 2018

Published online: 27 July 2018

\section{References}

1. Kim, S. H. et al. Characterization of ARD1 variants in mammalian cells. Biochem. Biophys. Res. Commun. 340, 422-427 (2006).

2. Chun, K. H. et al. Differential regulation of splicing, localization and stability of mammalian ARD1235 and ARD1225 isoforms. Biochem. Biophys. Res. Commun. 353, 18-25 (2007).

3. Chen, D. et al. daf-31 encodes the catalytic subunit of $N$ alphaacetyltransferase that regulates Caenorhabditis elegans development, metabolism and adult lifespan. PLoS Genet. 10, e1004699 (2014).

4. Ingram, A. K., Cross, G. A. \& Horn, D. Genetic manipulation indicates that ARD1 is an essential N(alpha)-acetyltransferase in Trypanosoma brucei. Mol. Biochem. Parasitol. 111, 309-317 (2000).

5. Ree, R. et al. The N-terminal acetyltransferase Naa10 is essential for zebrafish development. Biosci. Rep. 35, e00249 (2015). pii.

6. Wang, $Y$. et al. Drosophila variable nurse cells encodes arrest defective 1 (ARD1), the catalytic subunit of the major N-terminal acetyltransferase complex. Dev. Dyn. 239, 2813-2827 (2010).

7. Asaumi, M. et al. Interaction of N-terminal acetyltransferase with the cytoplasmic domain of beta-amyloid precursor protein and its effect on A beta secretion. J. Biochem. 137, 147-155 (2005).

8. Ohkawa, N. et al. N-acetyltransferase ARD1-NAT1 regulates neuronal dendritic development. Genes Cells 13, 1171-1183 (2008).

9. Seo, J. H. et al. ARD1-mediated Hsp70 acetylation balances stress-induced protein refolding and degradation. Nat. Commun. 7, 12882 (2016).

10. Shin, S. H. et al. Arrest defective 1 regulates the oxidative stress response in human cells and mice by acetylating methionine sulfoxide reductase A. Cell Death Dis. 5, e1490 (2014).

11. Lee, M. N. et al. Roles of arrest-defective protein 1(225) and hypoxia-inducible factor 1alpha in tumor growth and metastasis. J. Natl Cancer Inst. 102, 426-442 (2010).

12. Midorikawa, Y. et al. Identification of genes associated with dedifferentiation of hepatocellular carcinoma with expression profiling analysis. Jpn J. Cancer Res. 93, 636-643 (2002).

13. Ren, T. et al. Generation of novel monoclonal antibodies and their application for detecting ARD1 expression in colorectal cancer. Cancer Lett. 264, 83-92 (2008).

14. Yu, M. et al. Correlation of expression of human arrest-defective-1 (hARD1) protein with breast cancer. Cancer Invest. 27, 978-983 (2009).

15. Shim, J. H. et al. Clinical implications of arrest-defective protein 1 expression in hepatocellular carcinoma: a novel predictor of microvascular invasion. Dig. Dis. 30, 603-608 (2012).

16. Lee, D. et al. THU-104 - Close association of arrest-defective protein 1 (ARD1) overexpression with frequent postoperative recurrence and poor survival in patients with hepatocellular carcinoma. J. Hepatol. 66, S222 (2017).

17. Kang, H. J. et al. FRI-128 - overexpression of arrest-defective protein 1 in dysplastic hepatic nodule as a possible marker of malignant potential. J. Hepatol. 66, S468 (2017).

18. Whiteway, M. \& Szostak, J. W. The ARD1 gene of yeast functions in the switch between the mitotic cell cycle and alternative developmental pathways. Cell 43, 483-492 (1985).

19. Lee, F. J., Lin, L. W. \& Smith, J. A. N alpha acetylation is required for normal growth and mating of Saccharomyces cerevisiae. J. Bacteriol. 171, 5795-5802 (1989).

20. Park, E. C. \& Szostak, J. W. A. R. D. 1 and NAT1 proteins form a complex that has N-terminal acetyltransferase activity. EMBO J. 11, 2087-2093 (1992).

21. Arnesen, T. et al. Identification and characterization of the human ARD1-NATH protein acetyltransferase complex. Biochem J. 386, 433-443 (2005).

22. Arnesen, $\mathrm{T}$. et al. Induction of apoptosis in human cells by RNAi-mediated knockdown of hARD1 and NATH, components of the protein N-alphaacetyltransferase complex. Oncogene 25, 4350-4360 (2006).
23. Tribioli, C. et al. Isolation of new genes in distal Xq28: transcriptional map and identification of a human homologue of the ARD1 N-acetyl transferase of Saccharomyces cerevisiae. Hum. Mol. Genet. 3, 1061-1067 (1994).

24. $\mathrm{Xu}, \mathrm{H}$. et al. $\mathrm{N}$-alpha-acetyltransferase 10 protein inhibits apoptosis through RelA/p65-regulated MCL1 expression. Carcinogenesis 33, 1193-1202 (2012).

25. Yi, C. H. et al. A genome-wide RNAi screen reveals multiple regulators of caspase activation. J. Cell Biol. 179, 619-626 (2007).

26. Arnesen, T., Thompson, P. R., Varhaug, J. E. \& Lillehaug, J. R. The protein acetyltransferase ARD1: a novel cancer drug target? Curr. Cancer Drug Targets 8, 545-553 (2008).

27. Kalvik, T. V. \& Arnesen, T. Protein N-terminal acetyltransferases in cancer. Oncogene 32, 269-276 (2013).

28. Kuo, H. P. \& Hung, M. C. Arrest-defective-1 protein (ARD1): tumor suppressor or oncoprotein? Am. J. Transl. Res 2, 56-64 (2010).

29. Hua, K. T. et al. N-alpha-acetyltransferase 10 protein suppresses cancer cell metastasis by binding PIX proteins and inhibiting Cdc42/Rac1 activity. Cancer Cell 19, 218-231 (2011).

30. Wang, Z. et al. Inactivation of androgen-induced regulator ARD1 inhibits androgen receptor acetylation and prostate tumorigenesis. Proc. Natl Acad. Sci. USA 109, 3053-3058 (2012).

31. Zeng, Y. et al. Inverse correlation between Naa10p and MMP-9 expression and the combined prognostic value in breast cancer patients. Med. Oncol. 30, 562 (2013).

32. Seo, J. H. et al. Arrest defective 1 autoacetylation is a critical step in its ability to stimulate cancer cell proliferation. Cancer Res. 70, 4422-4432 (2010).

33. Vo, T. T. L. et al. ARD1-mediated aurora kinase A acetylation promotes cell proliferation and migration. Oncotarget 8, 57216-57230 (2017).

34. Zeng, Y. et al. Inhibition of STAT5a by Naa10p contributes to decreased breast cancer metastasis. Carcinogenesis 35, 2244-2253 (2014).

35. Seo, J. H. et al. Autoacetylation regulates differentially the roles of ARD1 variants in tumorigenesis. Int. J. Oncol. 46, 99-106 (2015).

36. Bilton, R. et al. Arrest-defective-1 protein, an acetyltransferase, does not alter stability of hypoxia-inducible factor (HIF)-1alpha and is not induced by hypoxia or HIF. J. Biol. Chem. 280, 31132-31140 (2005).

37. Park, J. H. et al. Nuclear translocation of hARD1 contributes to proper cell cycle progression. PLOS ONE 9, e105185 (2014).

38. Persson, B., Flinta, C., von Heijne, G. \& Jornvall, H. Structures of N-terminally acetylated proteins. Eur. J. Biochem. 152, 523-527 (1985).

39. Driessen, H. P., de Jong, W. W., Tesser, G. I. \& Bloemendal, H. The mechanism of N-terminal acetylation of proteins. CRC Crit. Rev. Biochem. 18, 281-325 (1985).

40. Hershko, A., Heller, H., Eytan, E., Kaklij, G. \& Rose, I. A. Role of the alpha-amino group of protein in ubiquitin-mediated protein breakdown. Proc. Natl Acad. SCi. USA 81, 7021-7025 (1984).

41. Hwang, C. S., Shemorry, A. \& Varshavsky, A. N-terminal acetylation of cellular proteins creates specific degradation signals. Science 327, 973-977 (2010).

42. Arnesen, T. et al. The chaperone-like protein HYPK acts together with NatA in cotranslational N-terminal acetylation and prevention of Huntingtin aggregation. Mol. Cell Biol. 30, 1898-1909 (2010).

43. Starheim, K. K., Gevaert, K. \& Arnesen, T. Protein N-terminal acetyltransferases: when the start matters. Trends Biochem. Sci. 37, 152-161 (2012).

44. Rope, A. F. et al. Using VAAST to identify an X-linked disorder resulting in lethality in male infants due to N-terminal acetyltransferase deficiency. Am. J. Hum. Genet. 89, 28-43 (2011).

45. Myklebust, L. M. et al. Biochemical and cellular analysis of Ogden syndrome reveals downstream Nt-acetylation defects. Hum. Mol. Genet. 24, 1956-1976 (2015).

46. Van Damme, P., Stove, S. I., Glomnes, N., Gevaert, K. \& Arnesen, T. A Saccharomyces cerevisiae model reveals in vivo functional impairment of the Ogden syndrome N-terminal acetyltransferase NAA10 Ser37Pro mutant. Mol. Cell Proteom. 13, 2031-2041 (2014).

47. Glozak, M. A., Sengupta, N., Zhang, X. \& Seto, E. Acetylation and deacetylation of non-histone proteins. Gene 363, 15-23 (2005).

48. Jeong, J. W. et al. Regulation and destabilization of HIF-1alpha by ARD1mediated acetylation. Cell 111, 709-720 (2002).

49. Lim, J. H., Chun, Y. S. \& Park, J. W. Hypoxia-inducible factor-1alpha obstructs a Wnt signaling pathway by inhibiting the hARD1-mediated activation of betacatenin. Cancer Res. 68, 5177-5184 (2008). 
50. Lim, J. H., Park, J. W. \& Chun, Y. S. Human arrest defective 1 acetylates and activates beta-catenin, promoting lung cancer cell proliferation. Cancer Res. $\mathbf{6 6}$ 10677-10682 (2006)

51. Shin, D. H., Chun, Y. S., Lee, K. H., Shin, H. W. \& Park, J. W. Arrest defective-1 controls tumor cell behavior by acetylating myosin light chain kinase. PLoS ONE 4, e7451 (2009)

52. Yoon, $\mathrm{H}$. et al. NAA10 controls osteoblast differentiation and bone formation as a feedback regulator of Runx2. Nat. Commun. 5, 5176 (2014).

53. Qian, X. et al. Phosphoglycerate Kinase 1 phosphorylates Beclin1 to induce autophagy. Mol. Cell 65, 917-931 (2017). e916.

54. Esmailpour, T. et al. A splice donor mutation in NAA10 results in the dysregulation of the retinoic acid signalling pathway and causes Lenz microphthalmia syndrome. J. Med. Genet. 51, 185-196 (2014).

55. Popp, B. et al. De novo missense mutations in the NAA10 gene cause severe non-syndromic developmental delay in males and females. Eur. J. Hum. Genet. 23, 602-609 (2015).

56. Rauch, A. et al. Range of genetic mutations associated with severe nonsyndromic sporadic intellectual disability: an exome sequencing study. Lancet 380, 1674-1682 (2012)

57. Sugiura, N., Adams, S. M. \& Corriveau, R. A. An evolutionarily conserved Nterminal acetyltransferase complex associated with neuronal development. J. Biol. Chem. 278, 40113-40120 (2003).

58. Kuo, H. P. et al. ARD1 stabilization of TSC2 suppresses tumorigenesis through the mTOR signaling pathway. Sci. Signal 3, ra9 (2010).

59. Yi, C. H. et al. Metabolic regulation of protein $\mathrm{N}$-alpha-acetylation by $\mathrm{BCl}-\mathrm{xL}$ promotes cell survival. Cell 146, 607-620 (2011).

60. Fisher, T. S., Etages, S. D., Hayes, L., Crimin, K. \& Li, B. Analysis of ARD1 function in hypoxia response using retroviral RNA interference. J. Biol. Chem. 280, 17749-17757 (2005)

61. Lee, E. J. et al. SAMHD1 acetylation enhances its deoxynucleotide triphosphohydrolase activity and promotes cancer cell proliferation. Oncotarget $\mathbf{8}$, 68517-68529 (2017)

62. Lee, C. F. et al. hNaa10p contributes to tumorigenesis by facilitating DNMT1mediated tumor suppressor gene silencing. J. Clin. Invest .120, 2920-2930 (2010).

63. Gromyko, D., Arnesen, T., Ryningen, A., Varhaug, J. E. \& Lillehaug, J. R. Depletion of the human Nalpha-terminal acetyltransferase A induces p53-dependent apoptosis and p53-independent growth inhibition. Int. J. Cancer 127, 2777-2789 (2010).

64. Park, J., Kanayama, A., Yamamoto, K. \& Miyamoto, Y. ARD1 binding to RIP1 mediates doxorubicin-induced NF-kappaB activation. Biochem. Biophys. Res. Commun. 422, 291-297 (2012)

65. Bosch, F. X., Ribes, J., Diaz, M. \& Cleries, R. Primary liver cancer: worldwide incidence and trends. Gastroenterology 127, S5-S16 (2004).

66. Tsuzuki, T. et al. Hepatic resection for hepatocellular carcinoma. Surgery 107, 511-520 (1990)

67. Okada, S. et al. Predictive factors for postoperative recurrence of hepatocellular carcinoma. Gastroenterology 106, 1618-1624 (1994).

68. Shin, J. W. \& Chung, Y. H. Molecular targeted therapy for hepatocellular carcinoma: current and future. World J. Gastroenterol. 19, 6144-6155 (2013)

69. Park, N. H., Song, I. H. \& Chung, Y. H. Chronic hepatitis B in hepatocarcinogenesis. Postgrad. Med. J. 82, 507-515 (2006).

70. Jang, M. K., Kim, H. S. \& Chung, Y. H. Clinical aspects of tumor necrosis factoralpha signaling in hepatocellular carcinoma. Curr. Pharm. Des. 20, 2799-2808 (2014).

71. Jin, Y. J. et al. Tumor necrosis factor-alpha gene polymorphism associated with development of hepatitis B virus-associated hepatocellular carcinoma. J. Clin. Gastroenterol. 49, e76-e81 (2015).

72. Lee, D. et al. Transforming growth factor beta 1 overexpression is closely related to invasiveness of hepatocellular carcinoma. Oncology 82, 11-18 (2012).

73. Song, B. C. et al. Association between insulin-like growth factor-2 and metastases after transcatheter arterial chemoembolization in patients with hepatocellular carcinoma: a prospective study. Cancer 91, 2386-2393 (2001).

74. Kim, S. H. et al. Prognostic value of serum osteopontin in hepatocellular carcinoma patients treated with transarterial chemoembolization. Korean J. Hepatol. 15, 320-330 (2009).
75. Lee, $\mathrm{H}$. et al. Overexpression of metastasis-associated protein 2 is associated with hepatocellular carcinoma size and differentiation. J. Gastroenterol. Hepatol. 24, 1445-1450 (2009).

76. Ryu, S. H. et al. Metastatic tumor antigen 1 is closely associated with frequent postoperative recurrence and poor survival in patients with hepatocellular carcinoma. Hepatology 47, 929-936 (2008).

77. Jin, Y. J. et al. Factors predisposing metastatic tumor antigen 1 overexpression in hepatitis B virus associated hepatocellular carcinoma. Dig. Dis. Sci. 57, 2917-2923 (2012)

78. Lee, D. et al. Safety and efficacy of adjuvant pegylated interferon therapy for metastatic tumor antigen 1-positive hepatocellular carcinoma. Cancer 119, 2239-2246 (2013).

79. Lee, S. H. et al. Single nucleotide polymorphisms associated with metastatic tumour antigen 1 overexpression in patients with hepatocellular carcinoma. Liver Int. 32, 457-466 (2012).

80. Ryu, S. H., Jang, M. K. Kim, W. J., Lee, D. \& Chung, Y. H. Metastatic tumor antigen in hepatocellular carcinoma: golden roads toward personalized medicine. Cancer Metastas. Rev. 33, 965-980 (2014).

81. Huang, G. W., Yang, L. Y. \& Lu, W. Q. Expression of hypoxia-inducible factor 1alpha and vascular endothelial growth factor in hepatocellular carcinoma: Impact on neovascularization and survival. World J. Gastroenterol. 11, 1705-1708 (2005).

82. Kim, K. R., Moon, H. E. \& Kim, K. W. Hypoxia-induced angiogenesis in human hepatocellular carcinoma. J. Mol. Med. 80, 703-714 (2002).

83. Chan, D. A., Sutphin, P. D., Yen, S. E. \& Giaccia, A. J. Coordinate regulation of the oxygen-dependent degradation domains of hypoxia-inducible factor 1 alpha. Mol. Cell Biol. 25, 6415-6426 (2005).

84. Clifford, S. C. et al. The pVHL-associated SCF ubiquitin ligase complex: molecular genetic analysis of elongin B and C, Rbx1 and HIF-1alpha in renal cell carcinoma. Oncogene 20, 5067-5074 (2001).

85. Huang, L. E., Pete, E. A., Schau, M., Milligan, J. \& Gu, J. Leu-574 of HIF-1alpha is essential for the von Hippel-Lindau (VHL)-mediated degradation pathway. J. Biol. Chem. 277, 41750-41755 (2002).

86. Kondo, K. et al. Inhibition of HIF is necessary for tumor suppression by the von Hippel-Lindau protein. Cancer Cell 1, 237-246 (2002).

87. Lee, J. W., Bae, S. H., Jeong, J. W., Kim, S. H. \& Kim, K. W. Hypoxia-inducible factor (HIF-1)alpha: its protein stability and biological functions. Exp. Mol. Med. 36, 1-12 (2004).

88. Masoud, G. N. \& Li, W. HIF-1alpha pathway: role, regulation and intervention for cancer therapy. Acta Pharm. Sin. B 5, 378-389 (2015).

89. Choi, K. S., Bae, M. K., Jeong, J. W., Moon, H. E. \& Kim, K. W. Hypoxia-induced angiogenesis during carcinogenesis. J. Biochem. Mol. Biol. 36, 120-127 (2003).

90. Hong, S. S., Lee, H. \& Kim, K. W. HIF-1alpha: a valid therapeutic target for tumor therapy. Cancer Res. Treat. 36, 343-353 (2004).

91. Pakala, S. B. et al. TGF-beta1 signaling targets metastasis-associated protein 1, a new effector in epithelial cells. Oncogene 30, 2230-2241 (2011).

92. Thiery, J. P. Epithelial-mesenchymal transitions in tumour progression. Nat. Rev. Cancer 2, 442-454 (2002).

93. Arnesen, T. et al. Interaction between HIF-1 alpha (ODD) and hARD1 does not induce acetylation and destabilization of HIF-1 alpha. FEBS Lett. 579, 6428-6432 (2005).

94. Murray-Rust, T. A., Oldham, N. J., Hewitson, K. S. \& Schofield, C. J. Purified recombinant hARD1 does not catalyse acetylation of Lys532 of HIF-1alpha fragments in vitro. FEBS Lett. 580, 1911-1918 (2006).

95. Huang, G. L. et al. LOH analysis of genes around D4S2964 identifies ARD1B as a prognostic predictor of hepatocellular carcinoma. World J. Gastroenterol. 16, 2046-2054 (2010)

96. Jiang, B. et al. Peptide mimic isolated by autoantibody reveals human arrest defective 1 overexpression is associated with poor prognosis for colon cancer patients. Am. J. Pathol. 177, 1095-1103 (2010).

97. Liu, C. et al. Combined phenotype of 4 markers improves prognostic value of patients with colon cancer. Am. J. Med Sci. 343, 295-302 (2012).

98. Wang, Z. H. et al. Up-regulation of human arrest-defective 1 protein is correlated with metastatic phenotype and poor prognosis in breast cancer. Asian Pac. J. Cancer Prev. 12, 1973-1977 (2011).

99. Arnesen, $\mathrm{T}$. et al. Expression of $\mathrm{N}$-acetyl transferase human and human Arrest defective 1 proteins in thyroid neoplasms. Thyroid 15, 1131-1136 (2005). 